\title{
A Case Report of Gemcitabine-Induced Steatohepatitis Associated with Adjuvant Monotherapy for Pancreatic Adenocarcinoma
}

\author{
Maria Vershvovsky, MD and Dina Halegoua-De Marzio, MD
}

\begin{abstract}
INTRODUCTION
Drug induced liver injury [DILI] is the most common cause of fulminant liver failure in the Western hemisphere. There are databases logging the myriad of herbs, supplements and medications that can cause DILI. Gemcitabine, a cytotoxic chemotherapy used most commonly in lung and pancreaticobiliary malignancy, has been well described as causing cholestatic liver injury. There are several case reports remarking on fatal hepatic failure with gemcitabine. This case describes steatohepatitis, a pattern of liver injury not previously described with gemcitabine.
\end{abstract}

\section{CASE PRESENTATION}

This case involves a 51-year-old healthy Caucasian female who presented to her primary care physician with jaundice and was found to have a $3 \mathrm{~cm}$ pancreatic head mass. She underwent surgical resection and was found to have 2 of 17 lymph nodes positive for adenocarcinoma. Her postoperative course was uncomplicated, and eight weeks later she was started on adjuvant chemotherapy with single agent gemcitabine for stage IIB pancreatic adenocarcinoma. Imaging studies before beginning chemotherapy revealed two arterially enhancing lesions in the liver consistent with focal nodular hyperplasia [FNH]. The patient had a reported history of Gilbert syndrome. She underwent genetic testing which confirmed homozygosity of UDP- gluconyltrasnsferase1A1*28 (UGT) allele which is associated with Gilbert's syndrome. The patient completed six 28-day cycles of standard dose gemcitabine, specifically, $1000 \mathrm{mg} / \mathrm{m} 2$ on days 1, 8, and 15 of each cycle. Routine surveillance CT imaging obtained at the start of cycle 3 revealed new hepatic steatosis. The patient was continued on gemcitabine and repeat surveillance imaging obtained at the end of cycle 4 revealed severe diffuse hepatic steatosis.

\section{DIFFERENTIAL DIAGNOSIS}

The patient was referred to a hepatologist. Genetic, metabolic and autoimmune causes of fatty liver disease were excluded. Specifically, the patient had laboratory testing for alpha-1 antitrypsin deficiency, Wilson's disease, hemochromatosis, autoimmune hepatitis, primary biliary cirrhosis and diabetes. The patient did not drink alcohol and had a normal body mass index. Additionally, viral hepatitides were ruled out. A comprehensive review of her medications did not reveal any known hepatotoxic agents.

\section{OUTCOME AND FOLLOW UP}

Subsequently, the patient had a dedicated MRI of the abdomen which confirmed the CT findings. The previously reported benign $\mathrm{FNH}$ lesions remained unchanged. The patient underwent a percutaneous liver biopsy three weeks after completing chemotherapy with histopathology revealing steatohepatitis with a NASH CRN score of $5 / 8$ and fibrosis score of $0 / 8$. This is a standardized scoring system used among pathologists to define histopathologic changes in the liver. Throughout cycles 3-6, the patient's bilirubin peaked at $1.0 \mathrm{mg} / \mathrm{dl}$ and she had a mild elevation of her transaminase and alkaline phosphatase levels. With the exception of moderate nausea, the patient tolerated chemotherapy well.

Three months after completing chemotherapy, the patient had imaging revealing innumerable metastatic lesions in the liver. She declined further systemic chemotherapy and had switched institutions to enroll in an immunotherapy clinical trial. Unfortunately, the patient succumbed to her disease and died shortly after her metastases were discovered.

\section{DISCUSSION}

In our literature search, there have been few cases describing gemcitabine associated steatohepatitis. The primary mechanism by which drugs lead to steatohepatitis is by inducing mitochondrial toxicity and causing accumulation of lipid byproducts and reactive oxygen species. There have been several molecular pathways implicated in pathogenesis of steatohepatitis, including PPAR and CY2D6 mutation. Common chemotherapeutic agents well described to cause this pattern of injury are irinotecan and oxaliplatin. We believe that gemcitabine is the causative agent of nonalcoholic fatty liver disease [NAFLD] in this patient. Her NAFLD progression shows a clear temporal relationship with gemcitabine introduction and an exhaustive work up excluded other etiologies. We suspect her steatohepatitis would have resolved with discontinuation of 
gemcitabine. Gemcitabine, a pyrimidine antimetabolite, is not dependent on UGT for glucorindation of its metabolites. There have been no previously established links between Gilbert's syndrome and increased risk of hepatotoxicity with gemcitabine, as there have with other drugs, such as irinotecan.

\section{KEY POINTS}

We hypothesize that patients with Gilbert syndrome may be predisposed to the effect of gemcitabine hepatotoxicity. Therefore, we recommend exercising caution in this patient population and having a low threshold for discontinuation of gemcitabine if there is elevation in transaminases or radiographic changes on crosssectional imaging.

\section{REFERENCES}

1. Costa ML, Lima-Júnior RC, Aragão KS, Medeiros RP, Marques-Neto RD, de Sá Grassi L, Leite LL, Nunes LG, de Mesquita Neto JW, de Castro Brito GA, de Souza MH, de Almeida PR, Ribeiro RA. Chemotherapy-associated steatohepatitis induced by irinotecan: a novel animal model. Cancer Chemother Pharmacol. 2014 Oct; 74(4):711-20.

2. Day CP, James OF. Steatohepatitis: a tale of two "hits"? Gastroenterology 1998 Apr;114(4):842-5.

3. Dobbie M, Hofer S, Oberholzer M, Herrmann R. Veno-occlusive disease of the liver induced by gemcitabine. Ann Oncol. 1998 Jun;9(6):681

4. Okada T, Egawa S, Motoi F, Yamamoto K, Ottomo S, Sakata N, Rikiyama T, Katayose Y, Unno M. Severe cholestatic liver failure associated with gemcitabine adjuvant monotherapy for pancreatic cancer. Clin J Gastroenterol. 2011 Dec;4(6):391-5.

5. Ren XS, Harada K, Yoshikawa S, Igarashi S, Urabe T, Yamada S, Katayanagi K, Kurumaya H, Nakanuma Y. A fatal case of progressive steatohepatitis, possibly chemotherapy-associated steatohepatitis related to gemcitabine. Clin J Gastroenterol. 2010 Aug:3(4):191-4.

6. Robinson K, Lambiase L, Li J, Monteiro C, Schiff M. Fatal cholestatic liver failure associated with gemcitabine therapy. Dig Dis Sci. 2003 Sep;48(9):1804-8

7. Tanaka H, Takamori H, Eto S, Ozaki N, Akaboshi S, Nakahara O, Ida S, Furuhashi S, Abe S, Horino K, Beppu T, Baba H. Acute liver injury with hepatic encephalopathy associated with gemcitabine administration for adjuvant chemotherapy in an HBV carrier with pancreatic cancer. Gan To Kagaku Ryoho. 2010 Sep:37(9):1783-6.

8. Venook AP, Egorin MJ, Rosner GL, Hollis D, Mani S, Hawkins M, Byrd J, Hohl R, Budman D, Meropol NJ, Ratain MJ. Phase I and pharmacokinetic trial of gemcitabine in patients with hepatic or renal dysfunction: Cancer and Leukemia Group B 9565. J Clin Oncol. 2000 Jul;18(14):2780-7.

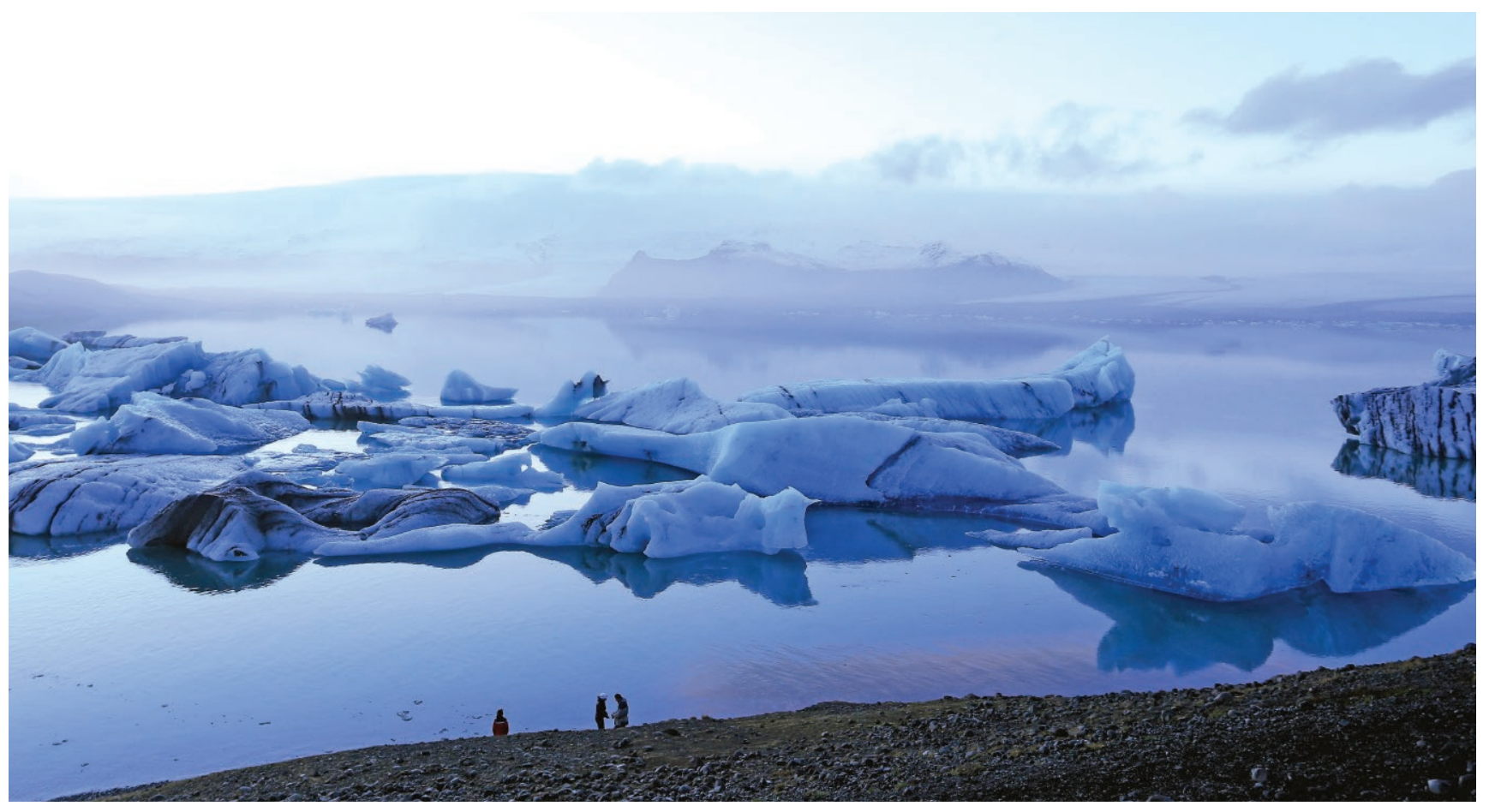

Sharon Li, MD 